\title{
PLANTAR ULCERS IN LEPROSY
}

\author{
Their Pathogenesis and Natural History, and their \\ Therapy and Prevention
}

by Johs G. Andersen, Cand. Med. et Chir. (Hafn)

(From the Hand Research Unit of Christian Medical College, Vellore under Prof. P. W. Brand; and the Schieffelin Leprosy.

Research Sanatorium, Karigiri under Dr. C. K. Joh.)

\section{SECTION 1. PATHOGENESIS ANI) NATURAL HISTORY (OF PLANTAR ULCERS.}

Perforating trophic ulcers of the sole of the foot were called "plantar ulcers" by PRICE, and we approve of this useful short term for this special condition. Plantar ulcers in leprosy constitute a major problem. The patient is disabled and often ostracized socially. The community loses a working member, who either turns to begging or becomes dependent on relatives. Often plantar ulceration is the condition which first brings the patient to seek medical relief. Unless we devise a simple, safe, and efficient method of therapy the patient will continue to suffer and our therapeutic clinics will be congested.

\section{The Normal Foot}

The normal foot has a highly specialised plantar surface with a high degree of resistance to trauma. The construction of this plantar surface comprises (1) a thick epidermis with a particularly thick cornified layer functioning very much like a car tyre on an automobile wheel; (2) a thick layer of subcutaneous fat caught under pressure in a mesh of tough fibrous strands which bind the skin and deeper layers together in a three-dimensional net which has a very great resilience both to vertical forces (pressure) and to horizontal forces (shear); (3) the bony and ligamentous structural base of the foot, which is reinforced with long tendons much in the manner of reinforced concrete. The foot at rest, the static foot, and the foot during take-off in walking; the kinetic foot, form two natural sections for consideration of the forces at play in the foot. In the static foot the total body weight is distributed more or less equally on the two soles. The heel takes one-fourth of the total body weight, and another fourth is taken evenly (NAPIER) by the 4 lateral metatarsal heads and the 2 sesamoid bones at the head of the first metatarsal. It is a simple pressure taken up by the sole and carried from the pressure points to the whole of the structural base of the foot. When the foot shares in propulsion of the body, a complex mechanism operates. To absorb and modify the jar of propulsion small 
movements take place in the skeletal structures from heel to metatarsal heads, and other adaptive changes in the structural base of the foot in relation to the subsurlace from which the propulsion lakes place. The pressure acts along the line from the heel to the metatarsal heads via the lateral horder of the loot. At the moment of thrust the total body weight bears on each of the 2 sesamoid bones. one-fourth, and on each of the 4 lateral metatarsal heads, one-eighth. As the metatarso phalangeal joints dorsiflex, the sesamoids move forward with the phalanges, and the first metatarsal head (which projects towards the sole) is pressed against the taut plantar fascia, and the amount of underlying tissue is much reduced. A similar process takes place at the lateral metatarsal heads. The metatarsal heads are thus relatively unprotected when they meet the final thrust (Hıcks).

The static loot meets pure pressure, the kinetic foot meets pressure plus shear. The amount of shear depends on the operation of a number of factors, namely the total body weight, the length of stride, the speed of propulsion, the nature and gradient of the ground surface, etc. The direction of the shear is longitudinal, transverse, and rotary, and the force is taken up and neutralised by the complex structure of the foot. It has been shown that the sites of choice of plantar ulcers correspond closely to the pressure points, more closely io the kinetic than the static (PRICI).

\section{Deviations from Normal in the Foot of a Leprosy Patient}

We shall consider these under the 3 main headings of anaesthesia, motor paralysis, and skeletal changes, and only in their relation to the natural history of plantar ulcers. Ancsesthesia of the foot in leprosy may be due either to widespread destruction of the fine termina! nerve fibres, or to neurofibrosis at a particular point of a bigger nerve, notably the posterior tibial, resulting in anaesthesia to touch, to pinprick, to heat and cold, sometimes with loss of the sense of vibration and position. Deep analgesia is less common. The extent and intensity vary. The foot has lost its warning mechanism. The patient is unaware of where and how he places his foot. He does not preceive traumata. The foot is therefore frequently put to harder use, and may be used in spite of lesions that would have stopped a normal person from using his foot. Anaesthesia is the central factor in the pathogenesis of plantar ulcers. Even in the absence of other pathological findings plantar ulcers are likely to develop. An anaesthetic foot with no other damage to its tissue is exposed to the risk of ulceration; it is "ulcer-liable". The moment ulceration takes place, the foot will have a permanent, stronger tendency to plantar ulceration; it will be "ulcer-prone". Even in the absence of actual ulceration. a scarred foot is ulcer-prone. 
The denervation of the foot in leprosy involves the sympathetic as well as sensory fibres. The area of anhydrosis is roughly the same as the area of anaesthesia. It is reasonable to suppose that the changes in vascular response might have some influence on the development of plantar ulceration. Experiments by FriTsCHI et al. and by ChATTERJEe, and recent observations of the digital arteries by JOB and ANDERSEN have not thrown any clear light on this problem.

Motor Paralysis. The motor paralysis in leprosy is selective and specific. In the vast majority only certain muscles are paralysed. This is in strict opposition to post-polio paralysis. Typically the ankle and toe dorsiflexors, the peronei and the intrinsic muscles are paralysed. The lack of the stabilising effect of the long-tendons may have a bearing on the development of tarsal instability. The specific consequences are dropfoot and clawtoes. A claw toe is cocked up in dorsiflexion at the metatarsophalangeal joint with the consequent sharp flexion of the interphalangeal joints. This has a marked influence on the windlass mechanism at the metatarsophalangeal joints (Hicks). It also takes the braking effect off the pulps of the toes and transfers it to the tips (PRICE). In severe clawing the tips of the toes are protected, while the metatarsal heads are subjected to a higher risk. There is general agreement that severe degrees of clawing should be corrected. It seems that even milder degrees of clawing are worth correcting.

The dropfoot in leprosy is typically a paralytic equinovarus foot with either drooping or clawed toes. The drooping foot will tend to drag along the ground. It will hit the ground first with the anterolateral border. It will not rest securely on the ground. The take-off thrust will tend to roll the foot over on the lateral metatarsal heads. An added ulcer risk is present at the antero-lateral border of the foot and at the tips of the dragging toes. The heel and the medial surface will be relatively protected.

Skeletal Changes. In the leprosy patient a number of skeletal changes of the foot are seen. Some are due to leprosy as a disease, others to incidental episodes (infection, scarring, loss of stability, etc.). The pathogenesis of these changes will not be discussed here. They will only be considered in their relation to development of plantar ulceration.

The stiff joints of a claw toe will have no particular effect except to make the claw deformity more vicious.

A stiff metatarsophalangeal joint, particularly at the first toe, will on the other hand seriously impair the take-off phase, leaving the foot either to take off in extremely high stepping or to place the final thrust on the very tips of the toes. This obviously adds considerably to the ulcer tisk at the tip of the toes.

In cases with phalangeal absorption the normal braking effect of 
the toes is lost. This exposes the metatarsal heads to more severe thrust, and will endanger this region. Concentric absorption of the metatarsal shafts will often lead to complete separation of the metatarsal heads (the term "pathological fracture" is a misnomer), with subsequent plantar displacement. The presence of bone close to the skin will add greatly to the risk of development of ulcers in this region.

Whatever the cause, any shortening of the foot will add to the amount of force that is brought to bear on a particular point, both in the static and in the kinetic foot. Even in the absence of particularly marked scarring, the short foot is a serious problem in ulcer prevention.

The so-called "neuropathy" of bone and joints in leprosy is not very well understood. Apparently it can be caused both by leprosy as such, by denervation, by infection, by scarring, and by any incidental trauma. Without entering into the discussion of the pathogenesis of "neuropathy" of bone and joints it can be stated, that any lack of stability, any deformation of structure, and any decrease in tissue vitality, will play a role in the development of plantar ulcers. Once these changes are established they are notoriously difficult to treat. A moderately flat foot, either a true pes plantis or a mild degree of eversion valgus deformity with tilting of the arch, is quite likely beneficial to the foot, since it tends to spread the weight bearing over a wider area. It also does away with the normal take-off thrust, the most dangerous part of the mechanism of the foot. In the more severe forms, especially the fully developed boat-shaped foot, the medial side of the foot is exposed to excessive danger, particularly at the navicular tuberosity, where very severe ulceration may develop.

\section{The Natural History of the Primary Ulcer}

It is the generally accepted impression in these two departments here that most plantar ulcers develop as what may be termed "closed ulcers" with only secondary breakdown into frank, open ulcers. It is well nigh impossible to obtain a reliable history of the ulcers. The basic pathology is the minimal necrosis blister or haematoma in the subcuticular or subcutaneous tissue, principally due to the shearing force of the kinetic foot. Many of these primary lesions undoubtedly heal up, but they leave behind them small stellate scars that will contract and jeopardize the blood supply. The general effect is creation of a vicious circle of haematoma-scar-haematoma. If unchecked the resilient weight bearing tissue will slowly be transformed into cicatricial, atrophic, ischaemic tissue with very little resistance to further traumata. A foot that presents its first open ulcer may very well have behind it a long history of a closed ulcer. 
In certain cases the haematoma will spread, particularly if it gets infected. Since the skin of the sole is very thick and tough, the blister or abscess cannot point to the immediate surface, but will spread like a cold abscess. It may wrap round the border of the foot or through the interdigital webs, and will present a larger daughter blister on the dorsum of the foot, as a wrap-round abscess. Occasionally the penetration goes deep and may involve the structural base of the foot, especially joint cavities. This collar-stud abscess may be combined with the wrap-round abscess.

In comparatively few cases a frank breach of continuity is the beginning of the ulcer process. It may be due to a nail in the shoe, a thorn, or a stone, or maybe a shoe bite. It may be an insignificant and unnoticed trauma that would have no such deleterious effect on a normal foot with preserved sensation. Whatever the cause, once ulceration starts, the vicious circle begins. The ulcer-liable foot has entered the ulcer-prone state.

Many patients wear shoes, either as part of their social environment or because they want to protect their feet. It is an unfortunate fact that most shoes available to these patients are not only without any protective value, but are directly harmful. Ideas of what a smooth in-sole is, what a good fit it is, and what damage nails can do, vary considerably from cobbler to physiotherapist.

If a shoe is worn in the presence of an open ulcer, the discharge, even the insignificant amount from a "dry ulcer", will wet the insole. From dust and discharge a roughness is gradually built up, that will be a constant source of irritation to the ulcer. Not infrequently a veritable cement pyramid sticking right up into the ulcer can be seen. So it might be better to avoid shoes altogether if you are not prepared to look after them properly and if you cannot get special shoes made by a specially trained cobbler. Corns and callosities are particularly likely to occur on anaesthetic feet. They have the same effect as a stone in the shoe. They are extremely dangerous, and should be avoided. If they do occur, they must be removed under the direction of a trained physiotherapist or surgeon.

The early, primary ulcer only involves the superficial tissues in the vast majority of cases. Rarely will a thorn penetrate right into an overlying joint cavity and start a primary ulcer with joint involvement. A primary ulcer has a comparatively strong tendency towards spontaneous healing. The factors that work against healing are the constant traumatising of the ulcer, and the inevitable infection. Of these infection is of less importance. The relative absence of infection in these open lesions exposed to dust and dirt is remarkable. It might be taken as a negative proof of the traumatic origin of plantar ulceration.

The habit of sitting cross-legged is responsible for the development of lateral malleolus ulcers. In spite of the position outside the 
plantar surface of the foot, their pathogenesis places them in the same group as the true plantar ulcers. They occur in an area with poor blood supply even in the normal individual. A major joint is very close. All in all they should be treated, with due respect as potentially very dangerous ulcers. They have a poor response to therapy.

\section{The Secondary Ulcer}

The basic pathology is the change from ulcer-liable to ulcer-prone state with the first introduction of ulceration and scar. Their natural history revolves round the vicious circle of scar-ulcer-scar. The important thing to keep in mind is that they are much more difficult to treat than primary ulcers. This alone points to prevention as the real answer to ulcer therapy.

\section{SECTION II. THERAPY AND PREVENTION OF PLANTAR ULCERS}

This section will attempt to describe an approach to the problems of a simple effective therapy for these ulcers, and effective prevention of them. Basically, the patients must be led to understand the factors involved, and to co-operate, and the same applies to the general public. There is a widespread false notion that plantar ulcers are an indication of infectivity, and the need is not understood for traditional trades to be adapted so as to be suitable for these patients, and they should be encouraged to re-enter the economic and social life of the community. An important fact to get accepted is that every anaesthetic foot is ulcer-liable, further that ulcer prevention is easier than cure and within the reach of most of these patients. Extra precautions must be taught thoroughly to a patient with an ulcer-liable foot. Because he has lost the normal warning mechanism of his foot, he must be taught the importance of thinking about the use of his feet. It is essential for the doctor or responsible person to conduct a daily inspection of the feet to detect evidence of even minor traumata, and the patient himself can do the same. He must also be taught care of the early, apparently insignificant lesions. He must understand the importance of adapting his daily life to this added risk. As far as possible standing and walking should be minimised, even if it involves adjustments of his work. The protective footwear will be dealt with later.

If an ulcer does occur, it must be treated immediately. The patient and the therapist must understand the importance of the "closed ulcers". If closed blisters and haematomas are treated with respect they deserve, much of the gross, really disabling ulceration can be avoided. 


\section{The Discharging Ulcer}

A frankly active uleer with discharge, slough, and undermined edges should be treated with strict bed-rest and elevation of the affected limb. Daily soaks in a solution of saturated magnesium sulphate or plain kitchen salt is the standard treatment. It should be followed by a simple dry dressing, mainly in order to keep flies off. but also for aesthetic reasons. Only rarely will one meet an uleer with frank lymphangiitis and general symptoms of sepsis, that presents an indication for treatment with systemic sulphonamides or antibiotics. Generally they should be avoided, since the systemically administered drugs have very little chance of reaching the organisms. embedded as they are in the necrotic tissue, hidden behind a solicl barrier of granulation tissue. The use of locally applied sulphonamides or antibiotics should be discouraged. They are not necessary. The chances of creating resistant strains of micro-organisms are very real. Plantar uleers are not due to leprosy as such. There is no indication for the use of specific anti-leprosy drugs in the treatment of ulcers.

Under this regime most ulcers dry up, or even heal with surprising speed.

\section{The Dry Ulcer}

This comprises both the ulcer that presents this stage when first seen and the discharging ulcer that has dried up under treatment. The logical treatment is strict bed-rest and elevation as described above. A dry dressing can be applied for aesthetic reasons. The lack of bed space, and also economic considerations have led to the adoption of a compromise. Application of a weil fitning below the knee plaster of paris hoot with a rocker device, preferably a Bohler walking iron is an excellent therapy. It is very important that a correct plaster technique be adopted. Padding of prominent bony points and exposed nerves is even more necessary than in a normal foot, since the anaesthesia makes development of pressure ulcers a very real risk. We prefer to leave the toes open for inspection. A toe guard is a good protection against traumata to the toes.

Most ulcers heal under this therapy within six weeks. The main advantage is that a number of patients can leave the hospital, and even take part in some light occupation during this period. The chief disadvantage is that it is unsuitable in a really wet climate. The actual cost of a plaster of paris boot with rocker is approximately the same as the cost of daily dressings for six weeks.

Corns. Treatment of corns is an essential part of ulcer therapy. The method of choice has until recently been surgical, entering the plane of cleavage with the pointed end of a pair of strong scissors. and separating the corn from the skin. Frischi now advocates 
soaking the foot for thirty minutes in a solution of sodium carbonate (four heaped spoonfuls of ordinary washing soda to the litre), after which the corn can be scraped off bluntly. If a haematoma or a necrosis blister is found under the corn, it should be treated as an ulcer.

Cracks. Frequently the sole of an anaesthetic foot develops cracks. Their sites of predilection are the bases of the toes, the sides of the heel, and the mid-foot. Often they penetrate deeply into the subcutaneous tissue, laying bare tendons, bone, or articular structures. As far as therapy is concerned they should be considered ulcers. They are often surrounded by hyperkeratotic cuticle, that should be saucerised to allow the crack to heal from the bottom.

Protective Footwear. An essential part of prevention in any ulcerliable or ulcer-prone foot is wearing of correct footwear. We have found that most of the shoes available to our patients are not only useless but directly harmful. Over several years work has been in progress to formulate the principles and practice of the correct footwear for anaesthetic feet. So far no reliable scientific method of assessing this problem has been developed. Recent experiments with pressure disc measurements (D. WARD and J. BAUMAN) seem to confirm the designs we have arrived at. In this paper the indications for the different types of shoe will be dealt with. A:brief description of the principles of the shoes will be given.

Three principles are essential. (1) Resilient insole to replace the lost or jeopardised resiliency of the planta; (2) Moulding to ensure a broader distribution of pressure; and (3) Rigidity to prevent angulation deformity in unstable feet, and particularly to change the thrust on special points during the take-off phase with a large amount of shearing force to a simple pressure take-off from the whole sole.

For the ulcer-liable foot and for the foot with very little scarring at the front part alone a simple sandal with resilient insole and tough undersole to prevent thorns from entering the insole is sufficient.

For the foot with some damage to the front part, but with no heel or mid-foot ulceration and with no damage to deeper structures, a metatarsal base bar must be added to the shoe. This should be placed well behind the damaged area, and not further forward than the mid-point of the foot (not the shoe). Its height should be as the heel. The effect of the bar is to shift the thrust further back, thus giving relative protection to the metatarsal head area, and to help in the moulding of the instep.

For the foot with extensive scarring, with damage to the deeper structures, with heel ulcers, with mid-foot ulceration, and for the shortened foot we recommend $a$ rigid sole with resilient insole and $a$ rocker. This shoe must have a full heel-cap to protect the heel from the dangerous lateral shear. The rocker should be placed at or 
slightly behind the mid-point of the foot. It should have the same height as the heel, or possibly slightly more. It causes the foot to take off from the rocker, distributing the total pressure over the whole area and diminishing the thrust on any one particular point. It is important that the tip of the shoe does not touch the ground during the take-off phase. When standing the patient must rest squarely on the rocker and the heel with no forward tilt of the shoe. The actual height of the rocker depends on the length of the stride and the length of the foot. An understanding patient who will take the trouble to educate himself to take short steps can get by with a much lower rocker. Moulding of the insole is a very tedious procedure. It is not very satisfactory under the rather poor conditions under which most of these shoes will have to be manufactured and worn. The adoption of microcellular rubher preferably of shore $15^{\circ}$ hardness, has not only given us an almost ideal resilience of the insole but has also permitted us to let the resilient insole take care of the moulding except in very special cases. The upper shoe is important too. Lack of really well trained cobblers and paucity of funds have forced us to adopt a very simple upper, much of the same type as the ordinary Indian sandal. The popular big-toe strap however is decidedly unsuitable for feet with intrinsic paralysis.

It must be impressed upon the patient that these shoes must be worn always, also inside the house and at the bathroom. This may go against the local custom but it is very important. Apart from simplicity of design and cheapness, the appearance of the shoe plays an important role. If we shall have any chance of success in our attempts to persuade the patients to wear these shoes, they must be made in such designs that they conform to local fashion as far as possible, and so that they do not stigmatise the patient as a "leper".

It is important to impress upon the patient, cobbler, physiotherapist and medical officer alike that shoes are preventive and not curative.

\section{Surgical Intervention in the Active Ulcer}

Only rarely will the surgeon be called upon to perform such operations. If simple, straightforward therapeutic measures in the treatment of ulcers as described above be adopted, most ulcers heal with surprising speed. There does not seem to be much difference between superficial and deep ulcers, provided open drainage is ensured. Very occasionally a sequestrum is formed that will have to be removed for healing to take place. One should be careful when diagnosing dead bone, since obviously naked bone has been seen to heal up. The radiological evidence of dead bone is not so conclusive in leprosy as in other diseases. Deep sinuous tracks, especially if leading to joint cavities, may have to be opened. Ulcers with under- 
mining edges and deep cracks may need the assistance of the surgeon. Simple procedures should be preferred, aiming at free drainage.

A heel ulcer may occasionally give rise to so widespread involvement of bone that surgical interference is indicated. DREISBACH advocates saucerising of offending bone through a fishmouth incision off the weight bearing surface. Occasionally it might be possible to combine this with excision of the ulcer and primary closure leaving the drainage to take place at the side of the heel.

Osteomyelitis of the sesamoid bones at the base of the first metatarsal bone is not infrequently an indication for surgical interference.

In all of these procedures it must be remembered that the operation is performed in actively infected tissue. Sufficient drainage is a sine qua non. The utmost conservatism should be the rule in the use of antibiotics and sulphonamides.

\section{Surgical Intervention in the Quiescent Phase}

When all ullceration and all residual heat and swelling have disappeared, the time has arrived for consideration of preventive surgery. As far as possible this should be done in an aseptic field. It is, however, well to remember that these operations will always have to be performed in potentially infected fields. The use of "antibiotic umbrellas" is strongly deprecated.

\section{Operations Aiming at the Correction of Paralytic Deformities}

Drop-foot and drop-toes should be corrected as soon as an aseptic field can be secured. It can be done in a single procedure without interfering with bone, which is a decided advantage. The method used by the author is recommended (circumtibial, subcutaneous transfer of the tibialis posterior tendon to the insertion of the tibialis anterior and subcutaneous transfer of the tendons of the extensor digitorum longus and extensor hallucis longus to the already transposed tibialis posterior tendon at the medial side of the leg.)

Claw toes can, if mobile, be corrected by a simple transfer of flexor tendon to the dorsal expansion. In cases of stiffness the offending joints should be resected. Occasionally claw toes are so bad that only amputation will do any good.

\section{Operations Aiming at the Correction of Skeletal Deformities}

The sequela of the deep ulcer, the adherent scar, is a menace to the foot. It is often an indication for removal of offending bone. The aim is to remove mechanically offending bone. The three 
common localities for this procedure are the heel, at the metatrasal heads, and the base of the fifth metatarsal bone. The operations should be done through a fish-mouth incision off the weight-bearing surface (DreisbaCH), Enough bone is sliced off or saucerised to give an even surface. The effect on the weight-bearing potential of the foot is of ten remarkable.

For extensive scarring of the fore-foot with a fairly well preserved hind-foot GASS recommended an amputation of the Lisfranc type with preservation of the largest amount of metatarsal shafts to allow a good flap from the plantar surface to be turned to the dorsum. This is undoubtedly a good procedure. Only it must be remembered that the field is almost certainly infected. Stiff, straight toes are frequently met with. They are highly offending and should either be amputated or "flailed" by resection of the offending joints.

Tarsal instability is an exceedingly troublesome condition. Its pathogenesis is not very well understood. Subtaloid arthrodeses have in many cases been attempted but with inconsistent results. The fixed tarsal deformities are equally important to correct. The methods employed here are modifications of Ryerson's, Dunn's and Lambrinudi's operations.

\section{Acknowledgments}

The author wishes to express his thanks to Dr. P. W. Brand, F.R.C.S. and to Dr. C. K. Job, M.D., and to colleagues at the two departments for valuable inspiration and criticism. Messrs. Bata and Co. have rendered help in obtaining the microcellular rubber for the shoes. The valuable support to departments from the Gandhi Memorial Leprosy Foundation and from the Indian Council of Medical Research is gratefully acknowledged.

\section{Summary}

In two papers an attempt has been made to present a concept of the natural history of plantar ulcers in leprosy with the main stress on factors dependent on anaesthesia of the foot. $\dot{A}$ rational therapy based on these findings has been described.

\section{Ref erences}

Brand, P. W. "Leprosy in Theory and Practice" (1958). Ed. by R. G. Cochrane, chapter XXI.

Chatterjee, S. N. "The Mechanism of Neural Signs and Symptoms in Leprosy" (1955). Internat. J. of Leprosy, 26, 1-17.

Chatterjee, S. N. “Mechanism of Blister Formation in Leprosy Patients" (1959). Internat. J. of Leprosy, 27, 305-312.

Dreisiach, G. "Leprosy in Theory and Practice" (1958). Ed. by R. G. Cochrane, chapter XXII.

FisCher, C. Leprosy Review (1955), $26,107$. 
FritsChI, E. P. Report to Indian Council of Medical Research on Enquiry into Circulation in the Feet in Leprosy Patients and into Methods of Prevention of Ulceration. (1958)

Fritschi, E. P. Report on the same Enquiry (under supervision of P. W. Brand). (1959)

Gass, H. H. "A Study of the Results of certain Surgical Procedures in Leprosy". (1938). Paper presented to the Cairo Conference.

HiCKS, J. H. "The Mechanics of the Foot" (1953). J. of Anat., 87, 345-357.

Hicks, J. H. "The Mechanics of the Foot" (1954). J. of Anat., 88, 25-30.

Napier, J. R. "The Foot and the Shoe" (1957). Physiotherapy, 43, 65-74.

Price, E. W. "Plantar Ulcers in Leprosy" (1959). Leprosy Review, 30, 98-105; 30, 180-183; 30, 242-248.

Sмiтн, J. W. "Muscular Control of the Arches of the Foot" (1954). J. of Anat., 88, $152-163$.

D. Wari \& J. Bauman. "Personal Communication". 